\title{
A Comparison of CDMA and Frequency Hopping in a Cellular Environment
}

\author{
Michael I. Mandell and Robert J. McEliece \\ California Institute of Technology, 116-81, Pasadena, UA 91125
}

\begin{abstract}
This paper compares the performances of Direct Sequence Code Division Multiple Access (CDMA) and Frequency Hopping (FH) schemes in a cellular multiuser environment. Our multiuser channel model incorporates the effects of propagation, frequency selective fading, and interference among users in the presence of a constrained system bandwidth. The CDMA and FH systems are compared using BPSK modulation. The main point of contrast between these systems is that the orthogonal hopping patterns in a FH system result in a decreased additive interference power, however the frequency spreading nature of CDMA results in the ability to combat fading. An information theoretic analysis is presented, which shows that system capacity is larger for CDMA than for FH. Hence, for this channel, with sufficient coding the CDMA system can achieve a higher level of performance than the FH system. However, it is unclear what level of complexity would be required to achieve such performance, and what effect such complexity would have on the practicality of the system.
\end{abstract}

\section{Summary}

In this paper we compare the performances of direct sequence Code Division Multiple Access (CDMA) and Frequency Hopping $(\mathrm{FH})$ in a cellular multiuser environment. We assume that there is a fixed system bandwidth, $B$, and a fixed data rate, $R$, at which each user communicates. The normalized traffic of a system, $p$, is defined to be the number of users per sector, $N_{s}$, divided by the ratio of $B$ to $R$. We find that the $\mathrm{FH}$ system sees less interference power than the CDMA system, however, the FH system is susceptible to frequency selective fades whereas the wide band nature of CDMA offers a level of diversity to such fading. Thus, a tradeoff in performance exists and the FH system performs better at higher levels of traffic with relatively high probability of bit error, and the CDMA system performs better at lower levels of traffic, with relatively low probability of bit error.

At this point, we consider the use of coding and present an information theoretic analysis. Assuming that there is no cooperation among the users in the system on the level of coding, the capacity of the system is defined to be the largest possible value of normalized traffic, $p$, for which each user in the system can communicate reliably at rate $R$. The capacity of FH and CDMA are computed and we find that the CDMA system has a larger capacity. This is due to the fact that the the FH system does not allow for the use of

* This work is supported by grants from GTE Laboratories and Pacific Bell, and AFOSR Grant 91-0037. very low code rates because using a low rate code a relatively small number of users would occupy the entire system bandwidth and thus result in a small amount of traffic. It turns out that, from an information theoretic viewpoint, that the ability of CDMA to use low rate codes is an advantage over the lower interference power in the FH system.

Since the information theoretic results are obtained over arbitrarily complicated coding schemes, and thus, arbitrarily long delays, we investigate the performance of specific coding schemes and the effects of a finite, controlled, delay using interleaving. These results are obtained primarily through simulation. Making some assumptions about the vehicle speed and transmitter frequency, we evaluate performance using a finite amount of interleaving delay by taking into account the actual amount of correlation among channel samples as seen from one codeword symbol to the next. We have evaluated the performance of several repetition codes as well as an $(8,4)$ bi-orthogonal block code. These coding schemes perform far below information theoretic capacity, and yield performance curves for $\mathrm{FH}$ and CDMA that cross with $\mathrm{FH}$ performing better higher levels of traffic with relatively high probability of bit error, and the CDMA performing better at lower levels of traffic, with relatively low probability of bit error.

\section{References}

[1] Wallace, Mark S., "High Capacity Digital Cellular Communications Through Slow Frequency Hopping CDMA", Proc. 29th Annual Allerton Conference on Communication, Control, and Computing,pg. 21, 1991.

[2] Verhulst, D., M. Mouley, and J. Szpirglas, "Slow Frequency Hopping Multiple Access for Digital Cellular Radiotelephone", IEEE Journal on Selected Areas in Communications, vol. SAC-2, no. 4 pp. 563-574, July 1984.

[3] Simon, M.K., J. K. Omura, R. A. Scholtz, and B. K. Levitt, "Spread Spectrum Communications", Vol. 2, MD: Computer Science Press, 1985.

[4] Jakes, W. C. Jr., "Microwave Mobile Communications", New York: Wiley, 1974.

[5] Proakis, John G., "Digital Communications", New York: McGraw Hill, 1989.

[6] Gilhousen, K. S., I. M. Jacobs, R. Padovani, A. J. Viterbi, L. A. Weaver, and C. E. Wheatley, "On The Capacity Of A Cellular CDMA System", IEEE Trans. Veh. Tech., vol. 40, no. 2, May 1991, pp. 303-312. 